\title{
Wikipédia, espace fluide, espace à parcourir
}

\section{Rémi Mathis}

\section{(2) OpenEdition}

\section{Journals}

\section{Édition électronique}

URL : https://journals.openedition.org/rbnu/2719

DOI : $10.4000 /$ rbnu. 2719

ISSN : 2679-6104

\section{Éditeur}

Bibliothèque nationale et universitaire de Strasbourg

\section{Édition imprimée}

Date de publication : 1 novembre 2012

Pagination : 52-61

ISSN : 2109-2761

\section{Référence électronique}

Rémi Mathis, "Wikipédia, espace fluide, espace à parcourir », La Revue de la BNU [En ligne], 6 | 2012 mis en ligne le 01 novembre 2012, consulté le 08 août 2021. URL : http://journals.openedition.org/ rbnu/2719; DOI : https://doi.org/10.4000/rbnu.2719

\section{(c) (i) (3)(2)}

La Revue de la BNU est mise à disposition selon les termes de la Licence Creative Commons Attribution - Pas d'Utilisation Commerciale - Partage dans les Mêmes Conditions 4.0 International. 


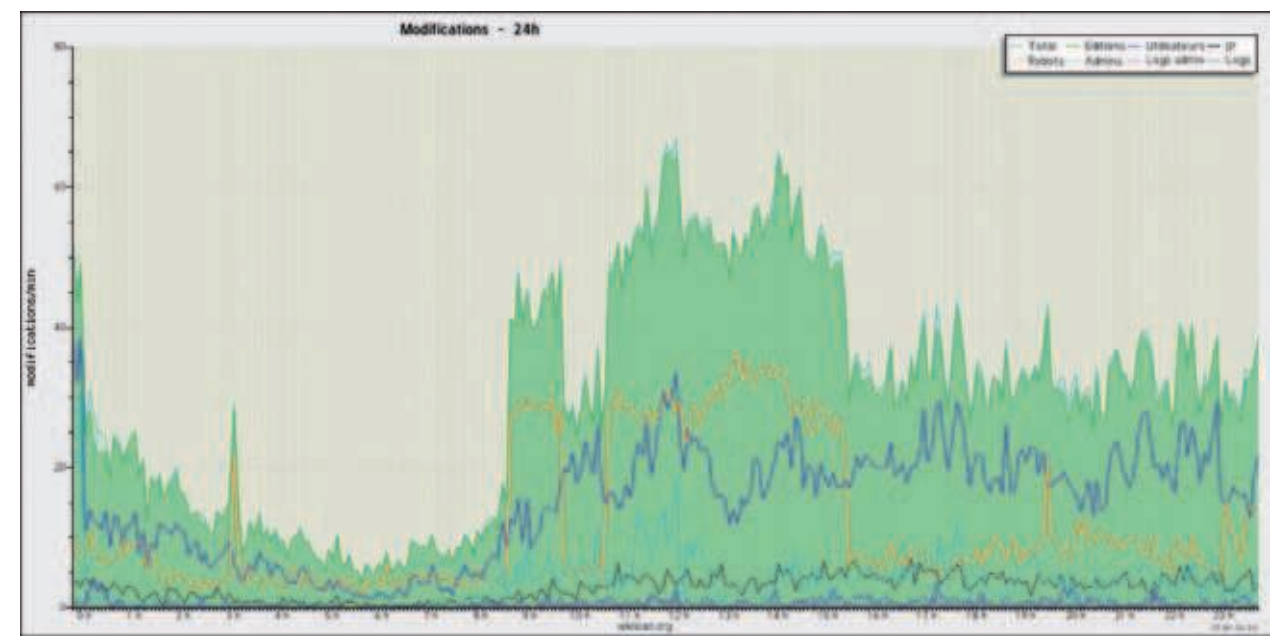

Répartition des contributions sur 24 heures

(23 août 2012)

\begin{tabular}{|c|c|c|c|}
\hline \multicolumn{3}{|c|}{ 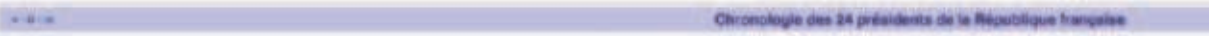 } & Whic: \\
\hline \multicolumn{3}{|c|}{ 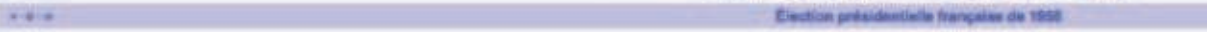 } & whiven \\
\hline \multicolumn{3}{|c|}{ 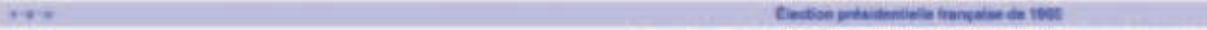 } & lathet \\
\hline \multicolumn{3}{|c|}{ 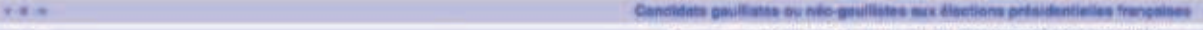 } & laven \\
\hline \multicolumn{3}{|c|}{ 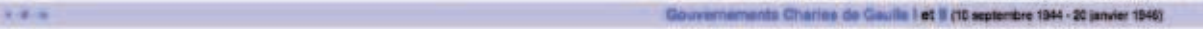 } & wint \\
\hline \multicolumn{3}{|c|}{ 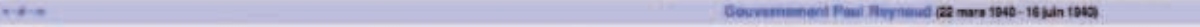 } & ithen \\
\hline \multicolumn{4}{|c|}{ 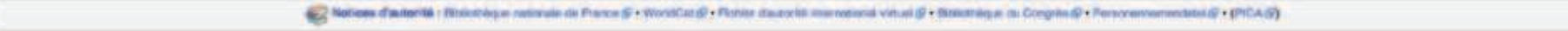 } \\
\hline \multicolumn{4}{|c|}{ 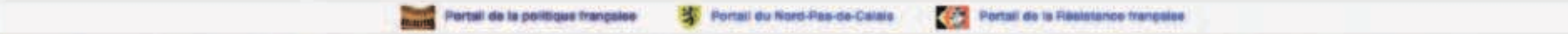 } \\
\hline \multicolumn{4}{|c|}{ 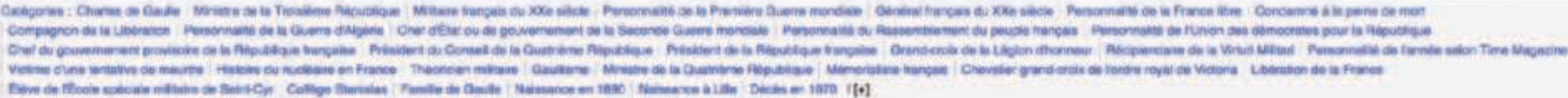 } \\
\hline
\end{tabular}

Catégories et boîtes

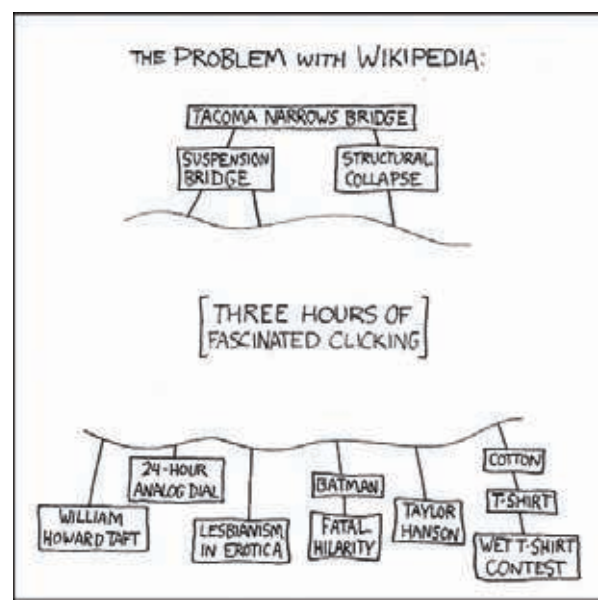

Randall Munroe, The problem with Wikipedia, in XKCD, $\mathrm{n}^{\circ} 214$ (xkcd.com) 


\section{Wikipédia, espace fluide, espace à parcourir}

Le nom de Wikipédia aujourd'hui est emblématique des nouvelles formes de construction et de diffusion du savoir rendues possibles par l'Internet. De l'encyclopédie, Wikipédia garde le projet universel, le rêve de totalisation, mais elle en subvertit la forme et l'autorité. En se substituant au livre imprimé, le média numérique permet l'actualisation et l'expansion infinies de l'encyclopédie, sa mise à jour en temps réel. En n'étant pas réalisée par un éditeur conventionnel, en n'étant pas contrôlée par un comité scientifique ou de rédaction, Wikipédia échappe au modèle centralisateur de l'édition de savoir traditionnelle, qui sépare sans communication possible les prescripteurs des consommateurs. Où se trouve le centre de Wikipédia? Il n'y a pas de centre, sinon les fermes de serveurs qui abritent les contenus. Wikipédia est un réseau en reconfiguration perpétuelle, organisé en aires linguistiques qui ne correspondent pas nécessairement aux découpages géographiques.

La présentation de Rémi Mathis ouvre un riche espace de réflexion et de questionnements pour tous ceux qui sont concernés par l'histoire des savoirs, leur validation sociale et leur devenir. Wikipédia déstabilise la hiérarchie des mondes savants, et fait de tout lecteur un contributeur potentiel. Elle fluidifie la fixation et la transmission des savoirs en affirmant leur perfectibilité, et fonde ainsi un espace public nouveau, un territoire partagé de la connaissance, où l'on cultive sa curiosité et son sens critique. Entre le livre et la bibliothèque, entre l'archive et le dictionnaire, évitant les écueils de la technicité elitiste comme de la vulgarisation condescendante, Wikipédia a inventé le premier média de référence du $21^{e}$ siècle.

\section{Christian Jacob}

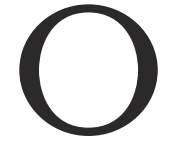

uvrez votre navigateur et rendez-vous sur le site suivant : http://lkozma.net/wpv/ index_fr.html.

Vous voyez en temps réel la localisation des contributions sur les wikipédias anglophone ou francophone. Il y en a en moyenne une par seconde sur la première et une toutes les trois secondes sur la deuxième. La localisation est approximative et indicative : elle se fonde sur l'adresse IP du contributeur (dont les caractères different selon la zone géographique où il se trouve). D'une seconde à l'autre, nous voici pris dans une folle farandole qui nous emmène de Bretagne en Provence, de Suède en Grèce, de Chine en Argentine à l'occasion d'ajouts d'informations sur les articles Usain Bolt, place Kléber, Écureuil roux... ou plusieurs millions d'autres. Les auteurs, eux, sont parfois désignés par l'adresse IP de leur ordinateur, parfois par le nom sous lequel ils ont décidé de s'inscrire, que ce soit un pseudonyme ou leur état civil officiel.
Toutes les données de Wikipédia sont librement lisibles par tous : cette transparence absolue est une des bases du fonctionnement d'un projet qui repose sur une communauté autogérée. Mais ces données sont généralement fournies sous forme de listes mises à jour en temps réel, qui sont très abstraites et ne donnent à la plupart des usagers qu'une bien mauvaise image de ce qui se passe réellement sur Wikipédia. Aussi cette simple application de " datavisualisation " qui situe dans le temps et l'espace toutes les contributions est-elle un outil particulièrement intéressant pour faire ressortir des phénomènes qui passeraient sans cela inaperçus.

Par sa taille gigantesque et son caractère foncièrement mouvant, Wikipédia est un excellent exemple des méthodes mises en œuvre pour représenter et mettre en espace les savoirs et ainsi les rendre plus accessibles. 


\section{Wikipédia comme espace virtuel, fluide et décentralisé}

Il n'existe pas une mais des wikipédias, chacune d'entre elles étant rédigée dans une langue différente. Certaines de ces langues correspondent à un territoire administratif unique (comme le hongrois, peu parlé en dehors de Hongrie) ou en tout cas bien circonscrit (le néerlandais est parlé aux Pays-Bas et en Belgique sous sa forme flamande; l'italien est parlé dans certains cantons suisses, etc.). D’autres wikipédias sont beaucoup plus internationales, soit que la langue en question soit parlée dans de nombreux territoires (c'est le cas du français, avec un nombre de locuteurs répartis dans toute la francophonie, bien au-delà des frontières de la France), soit qu'elle ne corresponde à aucun territoire du tout (la wikipédia en latin), soit que la langue soit tellement internationale que les locuteurs natifs sont loin d'être les seuls contributeurs (la wikipédia anglophone).

L'espace de Wikipédia est intrinsèquement un espace décentré, qui permet à la diversité des contributeurs, issus de tous les pays du monde, de se rencontrer en un lieu unique... qui n'existe pas. Ce lieu n'a en effet rien à voir avec le fait que la Wikimedia Foundation, qui gère le projet, soit implantée à San Francisco, ou que les serveurs sur lesquels se trouvent les données soient installés à Ashburn (Virginie) et à Tampa (Floride).

Wikipédia est un espace non seulement décentré mais également fluide, en perpétuelle mutation, rendant d'autant plus difficile son appréhension intellectuelle. Les articles de Wikipédia se veulent la synthèse de tous les savoirs disponibles dans le monde à un moment donné, l'état de l'art sur tous les sujets. Ses articles y sont forcément en évolution constante, dans la mesure où la production de ce savoir évolue elle-même rapidement. Chaque semaine paraissent des milliers d'articles scientifiques - parfois librement disponibles en " open access ", parfois sur abonnement - qui sont susceptibles d'être repris sur Wikipédia ${ }^{1}$. En dehors du savoir le plus légitime, de très nombreux articles sont directement en lien avec l'actualité, que ce soit la vie politique, le sport ou tout autre sujet (catastrophes naturelles, guerres, monde de l'art, etc.). Sur la wikipédia francophone, environ 800 ooo modifications ont lieu chaque mois. Bien que l'utilisation et la rédaction aient lieu à cette échelle planétaire, les contributions ne sont pas également réparties dans le temps : par exemple en début d'après-midi (heure française), on constate plus d'une modification chaque seconde... vandalisme compris (voir illustration p. 52)!

Voilà pourquoi les "wikipédiens " insistent sur le fait que Wikipédia n'est pas une encyclopédie mais un projet d'encyclopédie, un recueil d'articles dont l'étendue, la qualité, la longueur et le contenu même de chaque article évoluent constamment ; un objet en perpétuelle mutation dont la fluidité est voulue car elle est la condition sine qua non de son développement.

Tout est d'ailleurs organisé à la fois pour favoriser cette fluidité et pour l'apprivoiser. On la favorise en permettant à toute personne d'apporter sa pierre à l'édifice, sans avoir à décliner son identité ni même créer un compte. Ce n'est pas sans raison que "Wiki " signifie " vite " en hawaïen : la communauté s'est organisée afin d'éviter tout frottement, toute inertie, toute contrainte inutiles. Certes, les règles - votées par la communauté - sont extrêmement précises mais elles évoluent elles-mêmes au fur et à mesure des besoins et de l'identification de points d'achoppement. $\mathrm{Au}$ bout du compte, tout Wikipédia repose sur seulement cinq principes fondateurs dont le dernier est " Wikipédia n'a pas d'autres règles fixes ", avec son corollaire "N’hésitez pas!" (en anglais, " be bold !"). On apprivoise cette fluidité en s'assurant que chacune des milliers de contributions journalières est constructive. Toute assertion doit s'appuyer sur des sources préalablement publiées et l'espace de l'article est organisé pour mettre en valeur ses sources, présentées en notes de bas de page : l'espace du texte et l'espace des notes sont strictement séparés tout en se répondant et en étant intrinsèquement liés l'un à l'autre. Certains wikipédiens passent des heures parfois aidés par de petits programmes conçus pour l'occasion (des " bots ") - à scruter le journal des contributions (et plus encore des créations d'articles) afin de repérer le vandalisme, d'identifier l'assertion purement personnelle, de demander davantage de neutralité dans le traitement d'un sujet, de se plaindre de l'absence de source, de consulter la communauté sur la création de tel article qui ne pourra jamais respecter les règles (notamment de citations de sources tierces) du projet. Au-delà de l'activité spécifique de personnes particulièrement impliquées dans le projet, tous les wikipédiens qui possèdent un compte disposent d'une " liste de suivi ", les informant de toutes les modifications qui touchent des articles qu'ils ont eux-mêmes choisis. 


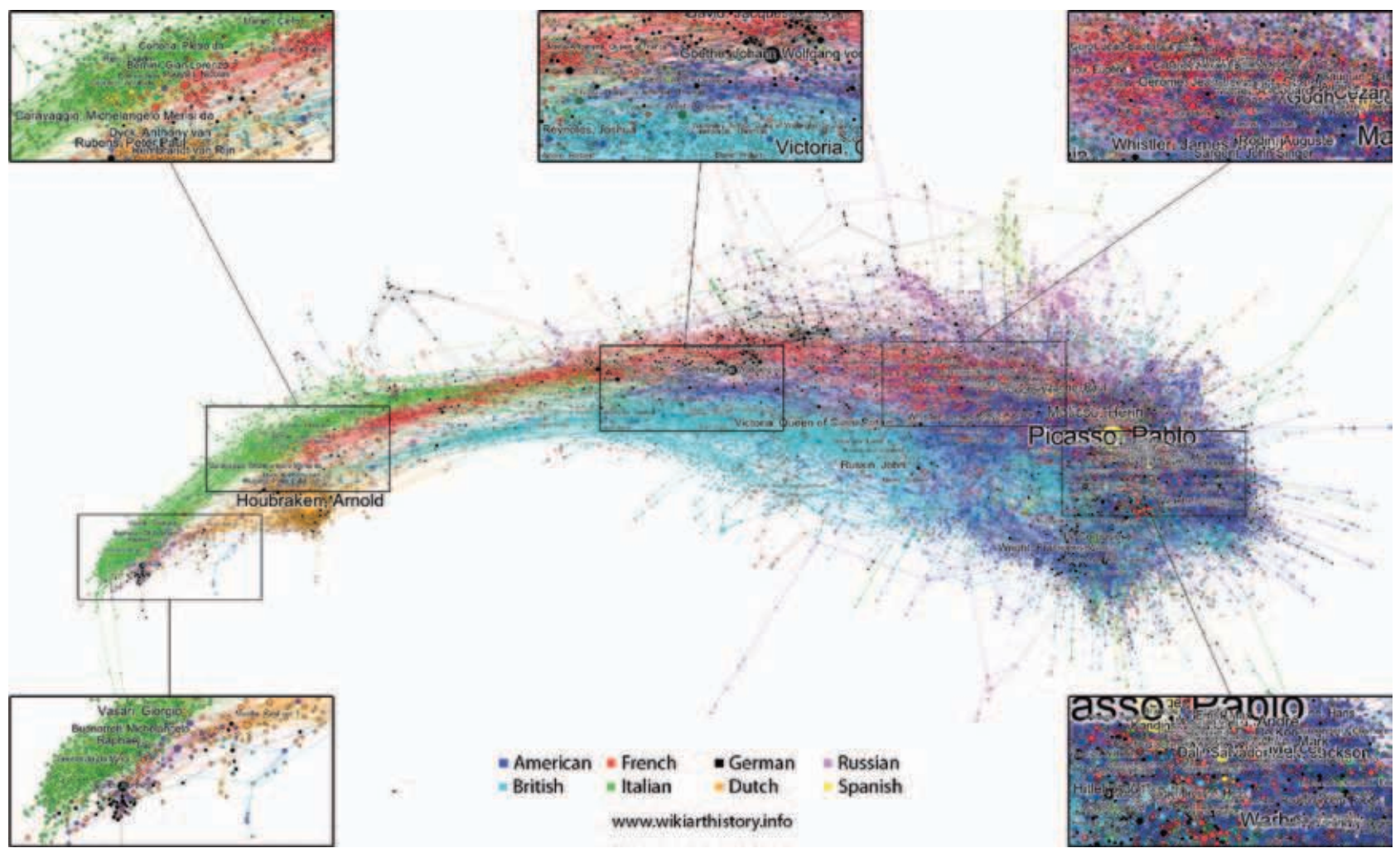

Représentations des liens entre articles consacrés à des acteurs de l'histoire de l'art dans la wikipédia anglophone, mai 2012,

Doron Goldfarb, Max Arends, Josef Froschauer, Dieter Merkl. 
En dernier recours, ce sera cependant toujours au lecteur de faire l'effort de naviguer dans ce monde en perpétuelle mutation. Contrairement à l'édition traditionnelle, Wikipédia affirme clairement qu'elle ne garantit pas la véracité des textes qu'elle offre ; on y récuse même le fait de chercher " la vérité ", à laquelle on préfère le fait de proposer une synthèse de l'état de l'art sur le sujet, reposant sur les sources disponibles à un moment donné, tout en étant conscients que ce savoir évoluera. Au lecteur, donc, d'apprendre à pratiquer une lecture intelligente et critique - celle du savant, du chercheur, et non la lecture passive de celui qui pense qu'une analyse est vraie parce qu'elle se trouve dans un livre, émise par un " intellectuel ". $\mathrm{Au}$ lecteur de comprendre comment ce système si fluide a abouti au texte qu'il peut lire, de se demander s'il s'agit d'une version stable ou s'il est tombé sur les quelques secondes où un acte de vandalisme n'a pas encore été décelé et réparé, de comprendre si l'article a été écrit par une ou trente personnes, s’il est le résultat de centaines d'heures de travail ou si personne ne s'y est réellement intéressé. Autant de réflexes qu'il faut acquérir et qui sont faciles à mettre en œuvre sur un site qui fait tout pour encourager cette lecture critique, notamment par sa transparence totale toute action est publique et toutes les archives sont partie intégrante du projet lui-même. On considère en effet que chaque modification - si modeste soit-elle sur un article crée une nouvelle version et chacune de ces versions est numérotée. Elle dispose même d'une URL unique et stable afin de pouvoir être citée : il devient dès lors possible de savoir exactement à quelle version d'un article on se réfère alors même que celuici change constamment. La question de la fluidité ne se pose cependant pas seulement à l'échelle de l'article. Naviguer dans l'espace décentralisé ainsi créé, s'y retrouver, accéder à des connaissances inconnues implique en effet que l'on dispose d'outils spécifiques.

\section{Des portes d'entrée pour naviguer, un espace structuré}

Chacune des wikipédias est divisée en plusieurs espaces. Le principal, appelé " main " ([espace] principal, en anglais), comprend les articles proprement dits. Mais Wikipédia étant un projet autogéré, il y a également beaucoup de pages qui servent à organiser le travail sur les articles. Un espace " discussion $"^{2}$ est intrinsèquement lié à " main " : chaque article possède son double dans " discussion " afin de permettre aux wikipédiens de discuter des améliorations à lui apporter et d'organiser le travail. Enfin, un espace communautaire appelé " Wikipédia " comprend toutes les pages d'aide, les rubriques permettant d'expliquer et d'analyser le projet ${ }^{3}$, sans lien direct avec les articles. L'espace de Wikipédia est ainsi organisé en sousespaces dont on sous-estime souvent l'importance. En août 2012, " main " (l'espace encyclopédique) représente moins du quart des pages de la wikipédia francophone ${ }^{4}$. Certains de ces sous-espaces sont directement utiles pour permettre au lecteur de se diriger dans ce magma de connaissances arrivant de partout et en perpétuelle mutation, grâce à des outils qui permettent de trouver ce que l'on cherche. C'est pour cela que les contributeurs de Wikipédia ont créé différentes formes de présentation de la connaissance. La plus simple est sans doute le portail thématique.

Il s'agit pour un thème donné de montrer de manière visuelle, dans des cadres de couleurs, la richesse des ressources proposées par Wikipédia en mettant en valeur les articles les plus importants, ainsi que ceux qui ont été reconnus de qualité supérieure par la communauté.

La plupart du temps, ces portails sont liés à un projet. On appelle projet un groupe de wikipédiens regroupés de manière informelle (le groupe pouvant lui-même évoluer au hasard de la disponibilité et de la motivation de ceux qui le composent) afin de traiter un thème de manière structurée et réfléchie. Il existe par exemple un projet Sport, un projet Escrime (qui est un sous-projet du projet Sport) ou un projet Sciences de l'information et des bibliothèques.

On y trouve entre autres des cadres qui proposent la liste des derniers articles créés sur le sujet, des principes de déclinaison visuelle des articles selon leur thème, un article du mois particulièrement mis en valeur. Non seulement les projets organisent les contenus afin qu'ils soient plus facilement accessibles, mais ils s'emploient également à évaluer l'avancement de l'article et son importance dans la thématique afin de définir des priorités d'action et de permettre aux lecteurs de mieux identifier ce qui est important ou ce qui l'est moins. Les portails relèvent en fait d'une éditorialisation des contenus, avec un véritable travail de médiation et de mise en valeur de la production par des contributeurs spécialisés.

La navigation est favorisée par d'autres outils intégrés 
aux articles eux-mêmes, qui constituent d'autres portes d'entrée dans la richesse de Wikipédia. Les catégories constituent un second moyen pour atteindre les articles recherchés et éviter de se perdre en route.

Chaque article possède plusieurs catégories, parfois un très grand nombre. Prenons l'exemple de w : sa vie bien remplie l'amène à être répertorié dans des catégories aussi diverses que " condamné à la peine de mort ", " théoricien militaire ", " mémorialiste français ", " naissance en 1890 ", " président du Conseil de la Quatrième République " ou encore " récipiendaire de la Virtuti Militari " (voir illustration p. 52). Ces catégories permettent de structurer toute information strictement factuelle, de la plus terre à terre à la plus fondamentale : on peut par exemple trouver rapidement tous les sénateurs à vie de la Troisième République, toutes les personnes célèbres nées à Besançon ou tous les graveurs français du $17^{\mathrm{e}}$ siècle. Des outils externes conçus et mis à disposition par des wikipédiens permettent de croiser ces catégories afin de préciser encore sa recherche.

S'en rapprochent un peu les boîtes qui se trouvent souvent en pied de page et proposent de manière organisée d'autres articles sur un même thème. Elles font l'objet d'une rédaction spécifique par des wikipédiens et elles sont intégrées par l'intermédiaire d'un modèle (" template "). Au pied de la page consacrée à l'université d'Oxford, on aura ainsi une boîte sur les universités au Royaume-Uni ou la Ligue européenne des universités de recherche. En pied d'article sur l'aluminium, une boîte en forme de tableau de Mendeleïev permet d'avoir accès à tous les éléments, et ainsi de suite.

Ce dispositif permet de signaler de manière optimale les articles à lire en priorité sur un sujet. L'exemple de Wimbledon montre qu'il s'agit aussi de fluidifier le parcours du lecteur, pour lui éviter de passer par un moteur de recherche à chaque fois qu'il veut faire évoluer sa lecture vers un article proche de celui qu'il est en train de lire (par exemple, passer des résultats de Wimbledon messieurs à ceux de Wimbledon dames; de ceux de Wimbledon 2012 à ceux de 2009, etc.) : il ne s'agit plus de considérer le savoir comme enfermé dans des articles clos sur eux-mêmes mais de permettre au lecteur de créer son propre parcours qu'il nous est impossible de déterminer par avance, puisqu'il est inventé au fil même de la lecture. Audelà d'une recherche ponctuelle de renseignements, si fine soit-elle, l'organisation du savoir doit permettre de faire des découvertes inattendues, de se perdre au hasard d'une navigation aisée et fluide, au fil de notre curiosité et de notre amour de la connaissance. Cette notion de sérendipité est centrale dans Wikipédia, car la taille de l'encyclopédie est telle qu'il est nécessaire de favoriser au maximum la circulation du lecteur afin de le faire profiter des richesses sans cesse renouvelées qu'elle contient.

\section{Favoriser la fluidité dans la lecture}

La circulation dans un espace tel que celui de Wikipédia repose avant tout sur les liens entre les articles.

Ce sont ces liens qui enrichissent infiniment l'expérience de lecture par rapport à une encyclopédie sur papier où les articles ne sont que juxtaposés, avec des renvois statiques et peu maniables. La capacité de passer directement d'un article à l'autre paraît maintenant si fondamentale que les encyclopédies traditionnelles choisissent de plus en plus de ne plus paraître que sous version électronique ${ }^{5}$. Comme tout ce qui apparaît sur Wikipédia, ces liens sont placés par les contributeurs, suivant des usages qui se sont développés dans la communauté, ce qui a permis l'émergence de recommandations. Bien sûr, créer des liens parmi 1300 ooo articles rend possible une infinité de parcours, condition nécessaire à tous les approfondissements comme au plaisir de l'errance (voir illustration p. 52)...

Ces liens dessinent une gigantesque toile d'araignée qui peut être représentée de manière visuelle. En partant du principe qu'un lien entre deux articles est le signe d'un lien intellectuel entre les sujets traités, ce sont ainsi de nouvelles connaissances qui peuvent être 


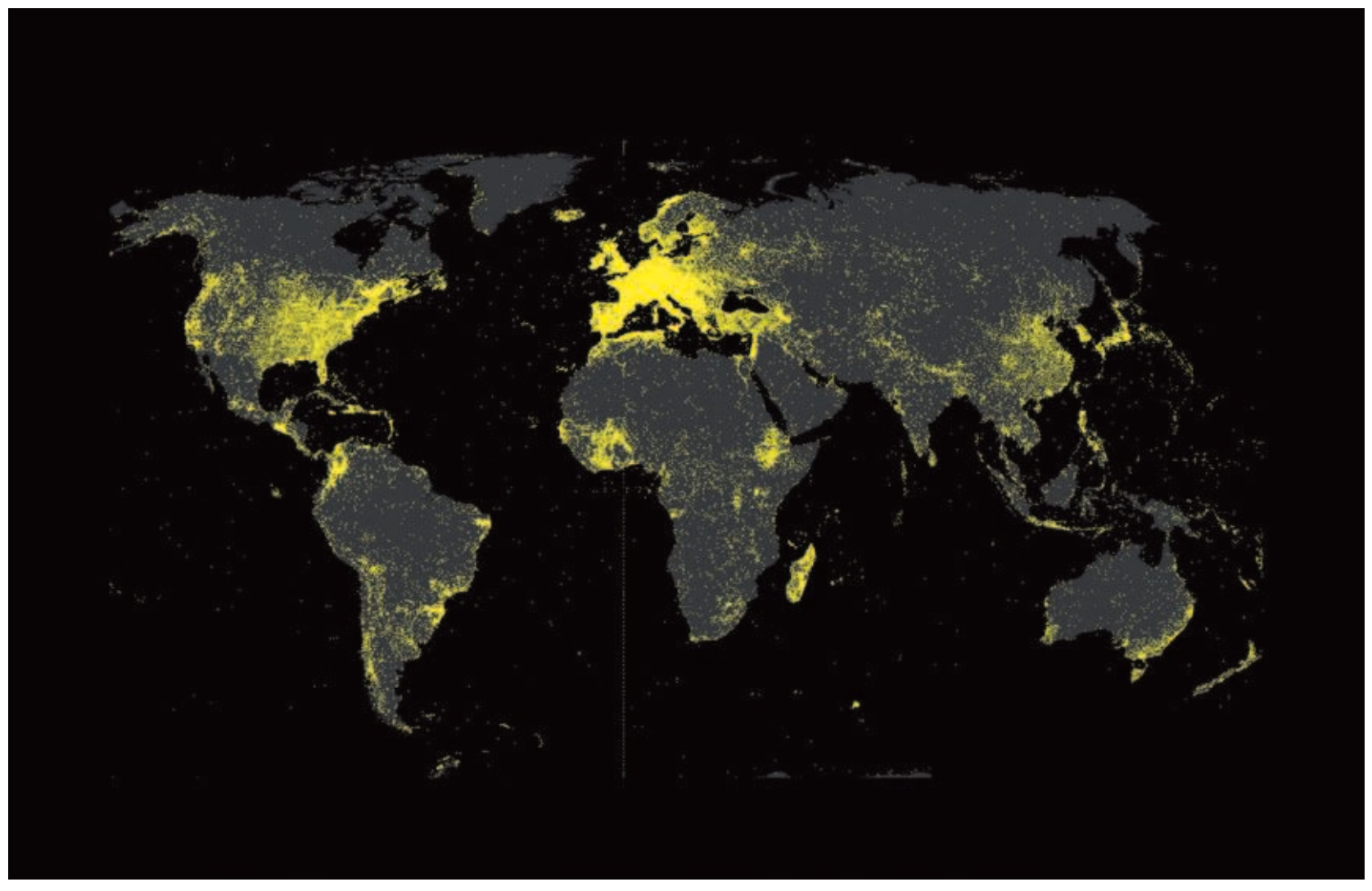

Articles « géotaggués " sur la wikipédia en français (novembre 2011). Mark Graham. 


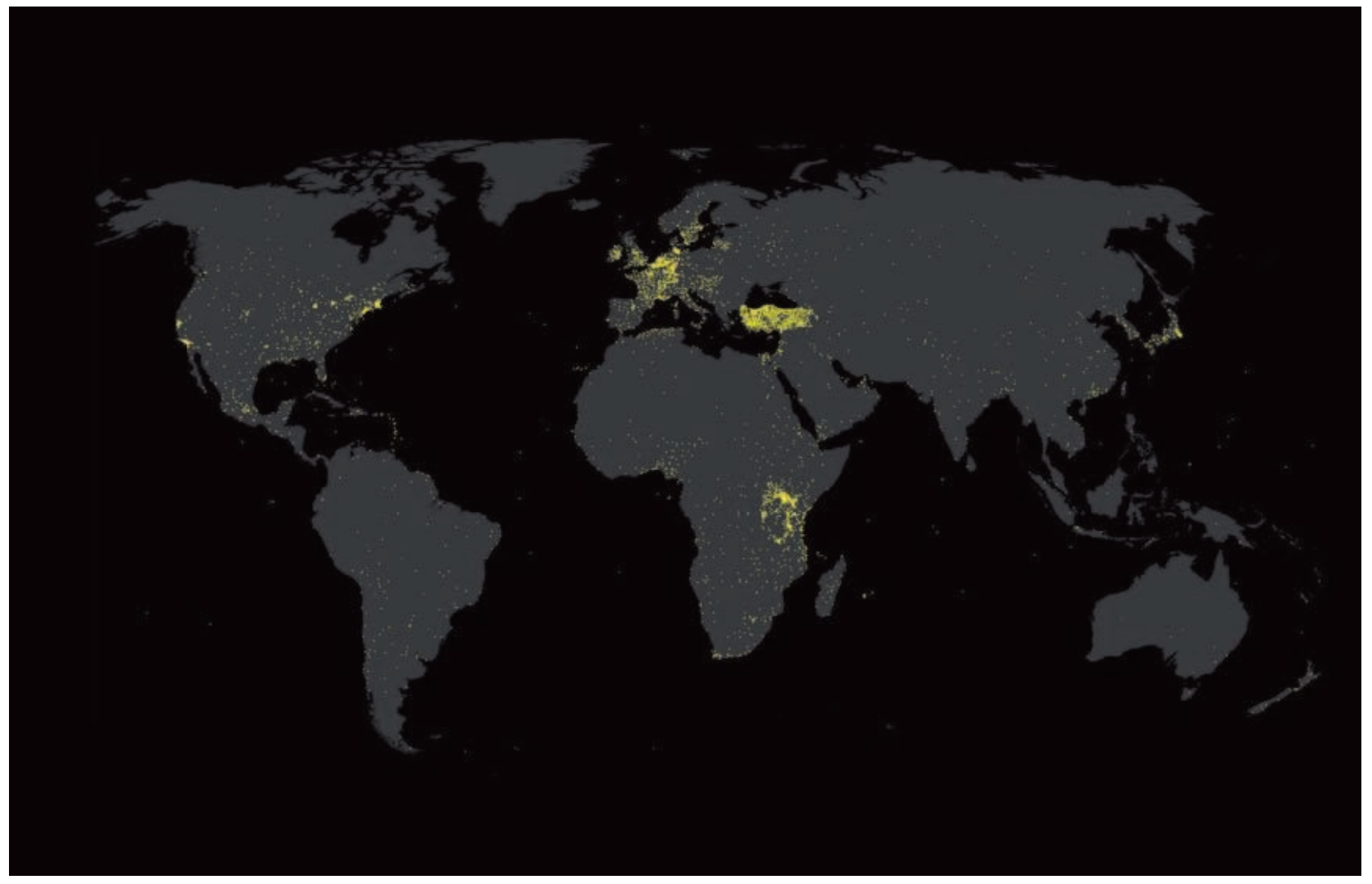

Articles " géotaggués " sur la wikipédia en swahili (novembre 2011). Mark Graham. 
tirées de la " datavisualisation ". Prenons un unique exemple : comment est représentée l'histoire de l'art dans Wikipédia? Quels liens entre artistes sont établis par les wikipédiens et quel paysage général se trouve ainsi déployé ? Y a-t-il plus d'articles sur l'art contemporain que sur l'art ancien ? À partir de quand l'art cesse-t-il d'être centré sur l'Europe ? La représentation dans Wikipédia reflète-t-elle bien les connaissances actuelles ? Des chercheurs ont réalisé une visualisation des liens entre les acteurs de l'histoire de l'art (séparés dans le temps par moins de 75 ans) : chacun des points possède une couleur qui correspond à une nationalité ${ }^{6}$ (voir illustration p. 55). On voit ainsi très bien les acteurs de la Renaissance italienne et flamande (en vert et ocre à gauche) peu à peu laisser place aux artistes français et anglais au cours des $17^{\mathrm{e}}-19^{\mathrm{e}}$ siècles, euxmêmes suivis par le post-modernisme américain dans un monde de l'art qui s'internationalise. N'oublions pas, bien sûr, que ceci n'est pas tant la réalité d'un phénomène historique que sa représentation dans la wikipédia anglophone : il serait intéressant de faire des comparaisons avec d'autres wikipédias mais aussi de manière diachronique. Changer la manière de représenter l'espace wikipédien et en donner un plan en deux dimensions permet de prendre du recul sur l'outil (et la pertinence de la connaissance qu'il diffuse) et de faire apparaître des phénomènes invisibles sans cela (voir illustration p. 55).

Au-delà des simples liens entre articles, une des originalités et un des grands avantages de Wikipédia repose sur le fait que la version francophone n'est qu'une parmi bien d'autres. Il y a en tout plus de 280 wikipédias dans toutes les langues.

Tout article de chaque Wikipédia est ainsi relié aux articles sur le même sujet dans toutes les langues. En fonction du nombre de ces "interwikis ", on peut avoir une idée de l'importance - ou en tout cas du caractère international d'un concept. C'est encore toute une géographie qui peut être représentée.

Certains articles sont internationaux par nature, comme par exemple les principales espèces animales, les concepts mathématiques ou les éléments chimiques. S'ils sont absents d'une langue, ce n'est que parce que le nombre d'articles dans cette langue est tellement réduit que personne n'a encore eu le temps de créer l'article sur la loutre ou le bismuth.

En revanche, la géographie devient plus signifiante dès qu'il s'agit de biographies, d'éléments historiques ou géographiques. Il est intéressant de constater que l'article sur le HMS Marlborough, navire britannique de la Première Guerre mondiale, existe (le 23 août 2012) en onze langues mais pas en français ; de même pour Katyn, village de Russie où eut lieu un célèbre épisode de la Seconde Guerre mondiale qui n'est pas traité en français alors qu'il l'est dans... 28 autres langues ! Comparer les wikipédias en différentes langues est ainsi un puissant moyen de comprendre les différences culturelles... mais aussi l'importance de Wikipédia pour les communautés linguistiques et la capacité de ces communautés à s'emparer d'un outil en fonction de leur taille, de leur accès à Internet et à la connaissance.

Les images des pages 58 et 59 partent des articles géolocalisés des wikipédias en français et en swahili et placent les points sur la carte du monde. Ce qui saute aux yeux est d'abord l'état d'avancement de chaque projet (la wikipédia en swahili compte 25 ooo articles seulement) mais aussi la prééminence des États-Unis et de l'Europe dans les deux cas. On remarque cependant que les articles sur le Kenya, l'Ouganda ou la Tanzanie sont davantage traités en swahili qu'en français, malgré la différence de taille des communautés.

Quand on quitte les sujets purement factuels pour entrer dans le traitement des concepts, l'établissement des liens dans Wikipédia s'avère plus problématique. Il est parfois difficile de relier des articles car les concepts ne se recoupent pas dans les deux langues. Le terme de " curator " en anglais signifie à la fois " conservateur " et " commissaire d'exposition ", ce qui est sujet à polémique en français. Si les conservateurs remplissent parfois la fonction de commissaires d'expositions, ils n'ont pas forcément une bonne opinion de ceux qui jouent ce rôle sans être employés par une institution : ils les soupçonnent d'agir en amateurs, voire d'usurper le prestige du titre pour des raisons commerciales ou de marketing. Lier l'article "Curator " sur la wikipédia anglophone à l'article "Conservateur " (et encore, lequel ? Il y a des articles distincts pour " conservateur de musée ", " conservateur d'archives " et " conservateur de bibliothèque ", sans parler du concept purement administratif et français de " conservateur du patrimoine ") ou "Commissaire d'exposition " est déjà un choix signifiant voire un sujet de discussions infinies. Il faut garder à l'esprit que toute association est le fruit d'un choix - rarement neutre - et, sur Wikipédia, ce choix peut être remis en cause à tout moment, suivant ce que la communauté pense être 
le plus pertinent. Cette capacité à faire circuler le lecteur dans un espace fluide ne se limite toutefois pas à Wikipédia, même en plusieurs langues. Les projets Wikimedia comprennent l'encyclopédie mais également bien d'autres ressources, également élaborées de manière collaborative et librement réutilisables. Parmi elles, on peut citer en particulier une base de données multimédia, Wikimedia Commons, ou une bibliothèque numérique, Wikisource.

En ce qui concerne les photographies ou les sons, certains sont directement insérés dans les articles de Wikipédia grâce à une ligne de code spéciale. On pourra ainsi écouter la Marseillaise ou voir des portraits de Victor Hugo sans quitter l'article qui leur est consacré.

Cependant, les ressources qui se trouvent sur les autres projets Wikimedia sont souvent d'une richesse telle qu'il est impossible, pour des raisons pratiques, voire non souhaitable, de toutes les faire figurer dans un article. Plusieurs centaines de photographies de la basilique Saint-Sernin de Toulouse sont disponibles dans Commons (classées dans des sous-catégories intérieur/extérieur/plans/portes/vitraux, elles-mêmes encore subdivisées), permettant de prendre connaissance de tous les détails de l'édifice. Qui consultera l'article sur Antoine Godeau, évêque de Vence au $17^{\mathrm{e}}$ siècle, pourra lire sur Wikisource des comptes rendus de ses visites paroissiales versés par les Archives des Alpes-Maritimes qui font travailler des apprentis paléographes à leur transcription.

Enfin, c'est grâce à un changement d'échelle et de technologie que l'on peut également se déplacer dans l'espace wikipédien, d'une manière appelée à être fondamentale pour l'avenir de Wikipédia et l'utilisation des données dans l'Internet de demain. De très nombreux sites et applications ont besoin de données sur tous les sujets. Pour cela toutefois, il faut posséder des informations structurées. Wikipédia propose de telles données, qui peuvent être réutilisées par tous. Elles ne sont toutefois pas livrées de manière brute, mais organisées et liées entre elles de manière signifiante : c'est ce qu'on appelle le "linked data " (données liées). Depuis 2007, l’Université libre de Berlin et celle de Leipzig, en collaboration avec l'entreprise OpenLink Software, travaillent sur les extractions de Wikipédia afin de proposer des ensembles de données structurées, de manière à prendre place dans ce nouveau paysage du web sémantique (c'est le projet DBpedia). Depuis peu, en France, l'INRIA, le ministère de la Culture et Wikimédia France ont lancé un projet : SemanticPedia. Le but est de se constituer en branche française de DBpedia et d'aller plus loin en travaillant sur d'autres projets tels que le Wiktionnaire?

Pour l'observateur extérieur, Wikipédia peut ressembler à un buisson foisonnant, en perpétuelle mutation et où il est difficile de se retrouver. Il n'est pas faux de dire qu'il s'agit en effet d'un objet complexe : on donne encore des cours à l'université sur son utilisation afin d'aider des jeunes gens de bon niveau à l'utiliser en toute connaissance de cause et de manière efficiente. Mais la fluidité de Wikipédia peut être aussi une force dans la mesure où la communauté est la première à ressentir les difficultés à appréhender l'objet. Des solutions sont ainsi trouvées en temps réel et mises en œuvre en même temps que se construit l'encyclopédie, que se rédigent les articles. Le support numérique et le caractère structuré d'une partie des informations sont à cet égard d'une grande aide, permettant de sans cesse reformater l'information, de la visualiser grâce à des outils externes et ainsi de proposer des outils d'aide à la lecture et à la recherche aussi fluides et évolutifs que les objets auquels ils sont liés.

\section{Rémi Mathis}

\section{Notes}

1 - Les liens vont d'ailleurs dans les deux sens puisque certaines revues comme $P L O S$ demandent désormais à leurs auteurs de préparer un résumé qui puisse directement servir à Wikipédia. Voir Rosemary Dickin, Bridging the Journal-Wikipedia Gap, PLoS blogs, 20 avril 2012, http://blogs.plos.org/plos/2012/04/bridging-the-journal-wikipedia-gap/

2 - Par exemple, discussion : Bibliothèque nationale et universitaire (Strasbourg) liée à l'article du même nom, http:|| fr.wikipedia.org/wiki/Discussion:Biblioth\%C $3 \%$ A8que_nationale_et_ universitaire_\%28Strasbourg\%29

3 - Par exemple, Wikipédia : Statistiques, http://fr.wikipedia.org/wiki/Wikip\%C3\%Agdia:Statistiques

4- Au 25 août 2012, 1288198 articles dans " main " pour 5263631 pages au total sur la wikipédia francophone.

5 - Voir Rémi Mathis, Non, Wikipédia n’a pas tué Britannica, in Libération, ecrans.fr, 20 mars 2012 ; http://www.ecrans.fr/Wikipedia-n-a-pas-tueBritannica,14292.html

6 - Doron Goldfarb, Max Arends, Josef Froschauer, Dieter Merkl, Art History on Wikipedia, a Macroscopic Observation, WebSci 2012, June 22-24, 2012

7 - Lancé en décembre 2002 par la Wikimedia Foundation, le wiktionnaire est un projet de dictionnaire participatif qui fonctionne selon les mêmes principes de base que Wikipédia ; voir http:/|fr.wiktionnary.org 